Nonlinear Processes in Geophysics (2005) 12: 75-81

SRef-ID: $1607-7946 / \mathrm{npg} / 2005-12-75$

European Geosciences Union

(c) 2005 Author(s). This work is licensed

under a Creative Commons License.

\title{
Modeling of short scale turbulence in the solar wind
}

\author{
V. Krishan ${ }^{1}$ and S. M. Mahajan ${ }^{2}$ \\ ${ }^{1}$ Indian Institute of Astrophysics, Bangalore 560 034, India \\ ${ }^{2}$ Institute for Fusion Studies, The University of Texas at Austin, Austin, Texas 78712, USA \\ Received: 6 August 2004 - Revised: 17 December 2004 - Accepted: 7 January 2005 - Published: 25 January 2005 \\ Part of Special Issue "Advances in space environment turbulence"
}

\begin{abstract}
The solar wind serves as a laboratory for investigating magnetohydrodynamic turbulence under conditions irreproducible on the terra firma. Here we show that the frame work of Hall magnetohydrodynamics (HMHD), which can support three quadratic invariants and allows nonlinear states to depart fundamentally from the Alfvénic, is capable of reproducing in the inertial range the three branches of the observed solar wind magnetic fluctuation spectrum - the Kolmogorov branch $f^{-5 / 3}$ steepening to $f^{-\alpha_{1}}$ with $\alpha_{1} \simeq 3-4$ on the high frequency side and flattening to $f^{-1}$ on the low frequency side. These fluctuations are found to be associated with the nonlinear Hall-MHD Shear Alfvén waves. The spectrum of the concomitant whistler type fluctuations is very different from the observed one. Perhaps the relatively stronger damping of the whistler fluctuations may cause their unobservability. The issue of equipartition of energy through the so called Alfvén ratio acquires a new status through its dependence, now, on the spatial scale.
\end{abstract}

\section{Introduction}

Solar wind is a turbulent supersonic outflow of plasma from the solar atmosphere. Fluctuations in the density,the velocity and the magnetic fields exist on several spatial and temporal scales. Transient disturbances such as those associated with Solar flare caused blast waves propagate out in the form of various linear and nonlinear plasma waves. Key Observations of the Solar Wind Turbulence have been summarized in Goldstein et al. (1994, 1995). The reduced Spectra of the fluctuations are obtained by averaging over the two directions perpendicular to the solar wind velocity $(V)$. The spectra are a function of the wavenumber along $V$. The spectral energy distributions of the velocity and the magnetic field fluctuations in the solar wind are now

Correspondence to: V. Krishan

(vinod@iiap.res.in) known in a wide frequency range, starting from much below the proton cyclotron frequency $(0.1-1 \mathrm{~Hz})$ to hundreds of $\mathrm{Hz}$. The inferred power spectrum of magnetic fluctuations consists of multiple segments - a Kolmogorov like branch $\left(\propto f^{-5 / 3}\right)$ flanked,on the low frequency end by a flatter branch $\left(\propto f^{-1}\right)$ and, on the high frequency end, by a much steeper branch $\left(\propto f^{-\alpha_{1}}, \alpha_{1} \simeq 3-4\right)$, (Coleman, 1968; Behannon, 1978; Denskat et al., 1983; Marsch, 1991; Leamon et al., 1998). Attributing the Kolmogorov branch $\left(\propto f^{-5 / 3}\right)$ to the standard inertial range cascade, initial explanations invoked dissipation processes (in particular, the collisionless damping of Alfvén and magnetosonic waves (Gary, 1999; Li et al., 2001), to explain the steeper branch ( $\left.\propto f^{-\alpha_{1}}, \alpha_{1} \simeq 3-4\right)$. However a recent critical study has concluded that damping of the linear Alfvén waves via the proton cyclotron resonance and of the magnetosonic waves by the Landau resonance, being strongly $\boldsymbol{k}$ (wave vector) dependent, is quite incapable of producing a power-law spectral distribution of magnetic fluctuations; damping mechanisms lead, instead, to a sharp cutoff in the power spectrum. Cranmer and Ballogoeijen (2003) have however, demonstrated a weaker than an exponential dependence of damping on the wave vector by including kinetic effects. However it is still steeper than that required for explaining the steepened spectrum.

An alternative possibility, suggested by Ghosh et al. (1996), links the spectral break and subsequent steepening to a "change" in the "controlling" invariants of the system in the appropriate frequency range. Matthaeus et al. (1996) have investigated the anisotropies in the spectral as well as in the variances of the 3-dimensional MHD turbulence. Stawicki et al. (2001) have invoked the short wavelength dispersive properties of the magnetosonic/whistler waves to account for the steepened spectrum and christened it as the spectrum in the dispersion range. In this paper we follow and develop these ideas within the frame work of Hall-MHD (HMHD). We will harness the three well-known invariants of HMHD (Mahajan and Yoshida, 1998; Krishan and Mahajan, 
$2005^{1}$ ). Using dimensional arguments of the Kolmogorov type, we will first derive the fluctuation spectra associated with the velocity and magnetic fields. We then go on to show that in different spectral ranges, different invariants control the energy cascade splitting the inertial range into distinct sections. The steeper and the flatter spectral branches (together with the standard branch), then, are all sub-parts of the extended inertial range. Invoking the hypothesis of selective dissipation, we then construct the entire magnetic spectrum with its three branches and two breaks by stringing together three spectral segments each controlled by one of the three invariants.

We briefly describe the nonlinear HMHD in Sect. 2. In Sect. 3, the respective spectral energy distributions are derived. The derived spectra are shown to account for the observed solar wind spectra in Sect. 4. The attempts invoking dissipation and dispersion are reviewed in Sect. 5. A short discussion and a summary of the conclusions constitutes Sect. 6.

\section{HMHD, nonlinear solution, invariants}

In the HALL-MHD comprising of the two fluid model, the electron fluid equation is given by

$m_{e} n_{e}\left[\frac{\partial \mathbf{V}_{e}}{\partial t}+\left(\mathbf{V}_{\mathbf{e}} \cdot \nabla\right) \mathbf{V}_{\mathbf{e}}\right]=-\nabla p_{e}-e n_{e}\left[\mathbf{E}+\frac{1}{c} \mathbf{V}_{\mathbf{e}} \times \mathbf{B}\right]$

Assuming inertialess electrons $\left(m_{e} \rightarrow 0\right)$, the electric field is found to be:

$\mathbf{E}=-\frac{1}{c} \mathbf{V}_{\mathbf{e}} \times \mathbf{B}-\frac{1}{n_{e} e} \nabla p_{e}$.

The Ion fluid equation is:

$m_{i} n_{i}\left[\frac{\partial \mathbf{V}_{\mathbf{i}}}{\partial t}+\left(\mathbf{V}_{\mathbf{i}} \cdot \nabla\right) \mathbf{V}_{\mathbf{i}}\right]=-\nabla p_{i}+e n_{i}\left[\mathbf{E}+\frac{1}{c} \mathbf{V}_{\mathbf{i}} \times \mathbf{B}\right]$

Substitution for $\mathbf{E}$ from the inertialess electron equation begets:

$m_{i} n_{i}\left[\frac{\partial \mathbf{V}_{\mathbf{i}}}{\partial t}+\left(\mathbf{V}_{\mathbf{i}} \cdot \nabla\right) \mathbf{V}_{\mathbf{i}}\right]=-\nabla\left(p_{i}+p_{e}\right)+\frac{1}{c} \mathbf{J} \times \mathbf{B}$

The magnetic induction equation becomes:

$\frac{\partial \mathbf{B}}{\partial t}=-c \nabla \times \mathbf{E}=\nabla \times\left(\mathbf{V}_{\mathbf{e}} \times \mathbf{B}\right)$,

where $\boldsymbol{B}$ is seen to be frozen to electrons. Substituting for $\mathbf{V}_{e}=\mathbf{V}_{i}-\mathbf{J} /$ en, one gets:

$\frac{\partial \mathbf{B}}{\partial t}=\nabla \times\left(\mathbf{V}_{i}-\frac{\mathbf{J}}{e n}\right) \times \mathbf{B}$.

We see that $\mathbf{B}$ is not frozen to the ions, $n_{e}=n_{i}=n$.

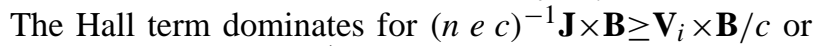
$L \leq M_{A} c / \omega_{p i}$ and $T \geq \omega_{c i}^{-1}$.

\footnotetext{
${ }^{1}$ Mahajan, S. M. and Krishan, V.: Exact Nonlinear Hall-MHD Waves, MNRAS, under submission, 2005.
}

That is the Hall term decouples electron and ion motion on ion inertial length scales and ion cyclotron times. Hall effect does not affect mass and momentum transport but it does affect the energy and magnetic field transport.

We present, here, an exact solution for Hall MHD (HMHD) allowing an ambient magnetic field which may be a local average in the case of the solar wind.

In the Alfvénic units with $B_{0}$ as the ambient field, $B_{0}=\widehat{e}_{s}$, $k_{s}=\boldsymbol{k} \cdot \widehat{e}_{s}$ is the projection of the wave vector along the direction of the field line, and $\perp$ is perpendicular to $\hat{e}_{s}$. Time and space variables are, respectively, measured in units of the ion gyroperiod $\omega_{c i}^{-1}=m_{i} c / e B_{0}$, and the ion skin depth $\lambda_{i}=c / \omega_{p i}$, where $\omega_{p i}=\left(4 \pi e^{2} n / m_{i}\right)^{1 / 2}$ is the ion plasma frequency. In the incompressible limit (plasma $\beta \rightarrow \infty$ ) we obtain the following dimensionless equations

$$
\begin{aligned}
& \frac{\partial \boldsymbol{B}}{\partial t}=\nabla \times[(\boldsymbol{V}-\nabla \times \boldsymbol{B}) \times \boldsymbol{B}] \\
& \frac{\partial(\boldsymbol{B}+\nabla \times \boldsymbol{V})}{\partial t}=\nabla \times[\boldsymbol{V} \times(\boldsymbol{B}+\nabla \times \boldsymbol{V})] .
\end{aligned}
$$

To look for wave like solutions' we split the fields into their ambient and the fluctuating parts (there is no ambient flow),

$\boldsymbol{B}=\widehat{e}_{s}+\boldsymbol{b} ; \quad \boldsymbol{V}=\boldsymbol{v}$

and substitute in Eqs. (7)-(8),

$$
\begin{gathered}
\frac{\partial \boldsymbol{b}}{\partial t}=\nabla \times\left[(\boldsymbol{v}-\nabla \times \boldsymbol{b}) \times \widehat{e}_{s}+(\boldsymbol{v}-\nabla \times \boldsymbol{b}) \times \boldsymbol{b}\right] \\
\frac{\partial}{\partial t}(\boldsymbol{b}+\nabla \times \boldsymbol{v})=\nabla \times\left[\boldsymbol{v} \times(\nabla \times \boldsymbol{v}+\boldsymbol{b})+\boldsymbol{v} \times \widehat{e}_{s}\right] .
\end{gathered}
$$

The nonlinear problem represented by Eqs. (10)-(11) is converted to a set of linear problems (the time honored method for solving nonlinear equations) by imposing the following conditions to eliminate the nonlinear terms:

$\boldsymbol{v}-\nabla \times \boldsymbol{b}=\alpha \boldsymbol{b}$

$\boldsymbol{b}+\nabla \times \boldsymbol{v}=\beta \boldsymbol{v}$,

where $\alpha$ and $\beta$ are like the separation constants. With the nonlinearities so taken care of, we are left with the remaining time dependent linear equations

$$
\begin{aligned}
& \frac{\partial \boldsymbol{b}}{\partial t}=\alpha \nabla \times\left[\boldsymbol{b} \times \widehat{e}_{s}\right] \\
& \frac{\partial}{\partial t}(\boldsymbol{v})=(1 / \beta) \nabla \times\left[\boldsymbol{v} \times \widehat{\boldsymbol{e}}_{s}\right] .
\end{aligned}
$$

Apparently we have traded a close nonlinear system (6 equations for six variables) for an overdetermined linear system (Eqs. 12-15) with 12 equations in six variables. Acceptable solutions, therefore, will be possible only under some particular conditions that will remove the over determination. To seek them, we first notice that Eq. (14) and Eq. (15) admit

$$
\begin{aligned}
\boldsymbol{b} & =\boldsymbol{b}_{k} \exp \left(i \underline{\mathrm{k}} \cdot \underline{\mathrm{x}}+i \alpha\left(\widehat{e}_{s} \cdot \underline{\mathrm{k}}\right) t\right) \\
\boldsymbol{v} & =\boldsymbol{v}_{k} \exp \left(i \underline{\mathrm{k}} \cdot \underline{\mathrm{x}}+i \frac{1}{\beta}\left(\widehat{e}_{s} \cdot \underline{\mathrm{k}}\right) t\right) .
\end{aligned}
$$


If the exponential solutions (Eq. 16) and (Eq. 17) are to satisfy the linear equations (12) and (13), we must require $\beta=1 / \alpha$. In addition, substituting Eqs. (16) and (17) into Eqs. (12) and (13) leads to

$$
\begin{gathered}
\boldsymbol{v}_{k}-i \underline{\mathrm{k}} \times \boldsymbol{b}_{k}=\alpha \boldsymbol{b}_{k}, \\
\boldsymbol{b}_{k}+i \underline{\mathrm{k}} \times \boldsymbol{v}_{k}=\frac{1}{\alpha} \boldsymbol{v}_{k},
\end{gathered}
$$

which, after simple manipulation, yield

$$
\begin{aligned}
& \boldsymbol{v}_{k}-\alpha \boldsymbol{b}_{k}=i \alpha \underline{\mathrm{k}} \times \boldsymbol{v}_{k} \\
& \boldsymbol{v}_{k}-\alpha \boldsymbol{b}_{k}=i\left(\underline{\mathrm{k}} \times \boldsymbol{b}_{k}\right) .
\end{aligned}
$$

Two consequences immediately follow:

$\boldsymbol{b}_{k}=\alpha \boldsymbol{v}_{k}$

relating $\boldsymbol{b}_{k}$ and $\boldsymbol{v}_{k}$, and

$\underline{\mathrm{k}} \times \boldsymbol{v}_{k}=-i \frac{1-\alpha^{2}}{\alpha} \boldsymbol{v}_{k}=-i \lambda \boldsymbol{v}_{k}$.

The first of these establishes the HMHD equivalent of the Alfvénic condition for MHD and the second, the Fourier transform of a Beltrami equation $(\nabla \times \underline{G}=\lambda \underline{G})$ has to be solved to complete the story; the solvability constraint will end up relating $\alpha$ with $k$ giving the "dispersion relation" $\alpha=\alpha(k)$.

The solutions of Eq. (23) are well-known and we could just quote them. But for completeness, we recapitulate a few steps in the process. Suppressing the indices for a simplified notation, we derive from Eq. (23): 1) dotting with $\boldsymbol{v}$ yields $\boldsymbol{v} \cdot \boldsymbol{v}=\boldsymbol{v}_{r}^{2}-\boldsymbol{v}_{i}^{2}+2 i \boldsymbol{v}_{r} \cdot \boldsymbol{v}_{i}=0$ implying $\left.\boldsymbol{v}_{i}=0 \pm \boldsymbol{v}_{r}, 2\right)$ and dotting with $\underline{\mathrm{k}}$ gives $\underline{\mathrm{k}} \cdot \boldsymbol{v}=0 \Rightarrow \underline{\mathrm{k}} \cdot \boldsymbol{v}_{r}=0=\mathrm{k} \cdot \boldsymbol{v}_{i}$. The suffix $r(i)$ denotes the real(imaginary) part. Crossing Eq. (23) with $\underline{\mathrm{k}}$ and using $\underline{\mathrm{k}} \cdot \boldsymbol{v}=0$, we obtain the dispersion relation (remembering that $\lambda$ is a function of $\alpha$ )

$\lambda= \pm k$.

Keeping track of the \pm may be notationally complicated. Since the physics is the same, we will investigate the option, $\boldsymbol{v}_{i}=\boldsymbol{v}_{r}$ and $\lambda=k$. For this choice, it is straightforward to show that $\widehat{\boldsymbol{v}}_{r}, \widehat{\boldsymbol{v}_{i}}$, and $\widehat{\widehat{\mathrm{k}}}$ form a right-handed orthogonal triad of unit vectors.

Let us first choose $\widehat{\boldsymbol{v}}_{r}, \widehat{\boldsymbol{v}}_{i}$, and $\widehat{\underline{\mathrm{k}}}$ to be $\widehat{e}_{x}, \widehat{e}_{y}$, and $\widehat{e}_{z}$, respectively. This choice dictates the following expressions for the velocity and the magnetic fields $\left(\underline{\mathrm{k}}=k \widehat{e}_{z}\right.$, and $A_{0}$ is a constant amplitude);

$\boldsymbol{b}=\alpha \boldsymbol{v}$,

$\boldsymbol{v}=A_{0}\left[\widehat{e}_{x}+i \widehat{e}_{y}\right] \exp \left(i k z+i \alpha k\left(\widehat{e}_{z} \cdot \widehat{e}_{s}\right) t\right)$

with $\alpha$ determined by

$$
\begin{aligned}
k=\lambda & =\frac{1-\alpha^{2}}{\alpha}, \\
\alpha_{ \pm} & =\left[-\frac{k}{2} \pm\left(\frac{k^{2}}{4}+1\right)^{1 / 2}\right] .
\end{aligned}
$$

From Eqs. (26) and (28), we extract the effective frequency of the circularly polarized wave,

$\omega_{ \pm}=-k\left[-\frac{k}{2} \pm\left(\frac{k^{2}}{4}+1\right)^{1 / 2}\right]\left(\widehat{e}_{z} \cdot \widehat{e}_{s}\right)$,

a result which is valid over a wide range of $k$ from $k \ll 1$ MHD end to the $k \gg 1$ Hall dominated regime. The $k$ dependence of the separation constant, implying a $k$ dependent relationship between $\boldsymbol{b}$ and $\boldsymbol{v}$ is one of the defining and distinguishing characteristics of the new broadband fully nonlinear incompressible wave. To make contact with the familiar, let us examine the two extreme limits of the general result. For $k \ll 1$,

$\alpha \rightarrow \pm 1, \omega \rightarrow \mp k\left(\widehat{e}_{z} \cdot \widehat{e}_{s}\right)$,

reproducing the $k$ independent MHD Alfvénic relationship for both the co- and the counter propagating waves. In the $k \gg 1$ regime, however,

$\alpha_{+} \rightarrow 1 / k, \alpha_{-} \rightarrow-k$,

with

$\omega_{+} \rightarrow-\widehat{e}_{z} \cdot \widehat{e}_{s}, \omega_{-} \rightarrow\left(\widehat{e}_{z} \cdot \widehat{e}_{s}\right) k^{2}$.

It is easy to recognize, in analogy with the linear theory, that the $(+)$ wave is the shear-cyclotron branch, while the $(-)$ represents the whistler mode. the frequency of the $(+)$ wave approaches some fraction of the ion gyro frequency (normalizing frequency) - it is only when $\underline{\mathrm{k}}$ and $\boldsymbol{B}_{0}$ are fully aligned $\left(\widehat{e}_{z} \cdot \widehat{e}_{s}= \pm 1\right)$ that the wave reaches the cyclotron frequency asymptotically. In this limit the magnitudes of the velocity and magnetic fields can vastly differ (they still remain aligned). The respective relationships are

$\boldsymbol{v} \rightarrow k \boldsymbol{b}$.

for the $(+)$ branch, and

$\boldsymbol{b} \rightarrow k \boldsymbol{v}$.

for the (-) branch; the compressional-whistler mode is dominated by the magnetic energy, while in the shear-cyclotron mode, the kinetic energy dominates.

The well-known invariants of the HMHD system (Mahajan and Yoshida 1998),

Total energy:

$E=\frac{1}{2} \int\left(\boldsymbol{v}^{2}+\boldsymbol{b}^{2}\right) d^{3} x=\frac{1}{2} \sum_{k}\left|v_{k}\right|^{2}+\left|\boldsymbol{b}_{k}\right|^{2}$

Magnetic helicity:

$H_{M}=\frac{1}{2} \int \mathrm{A} \cdot \mathrm{B} d^{3} x=\frac{1}{2} \sum_{k} \frac{i}{k^{2}}\left(\boldsymbol{k} \times \boldsymbol{b}_{k}\right) \cdot \boldsymbol{b}_{-k}$

Generalized helicity:

$$
\begin{aligned}
H_{G} & =\frac{1}{2} \int(\boldsymbol{A}+\boldsymbol{V}) \cdot(\boldsymbol{b}+\nabla \times \boldsymbol{v}) d^{3} x \\
& =\frac{1}{2} \sum_{k}\left[\frac{i \boldsymbol{k} \times \boldsymbol{b}_{k}}{k^{2}}+\boldsymbol{v}_{k}\right] \cdot\left[\boldsymbol{v}_{-k}-i \boldsymbol{k} \times \boldsymbol{v}_{-k}\right],
\end{aligned}
$$



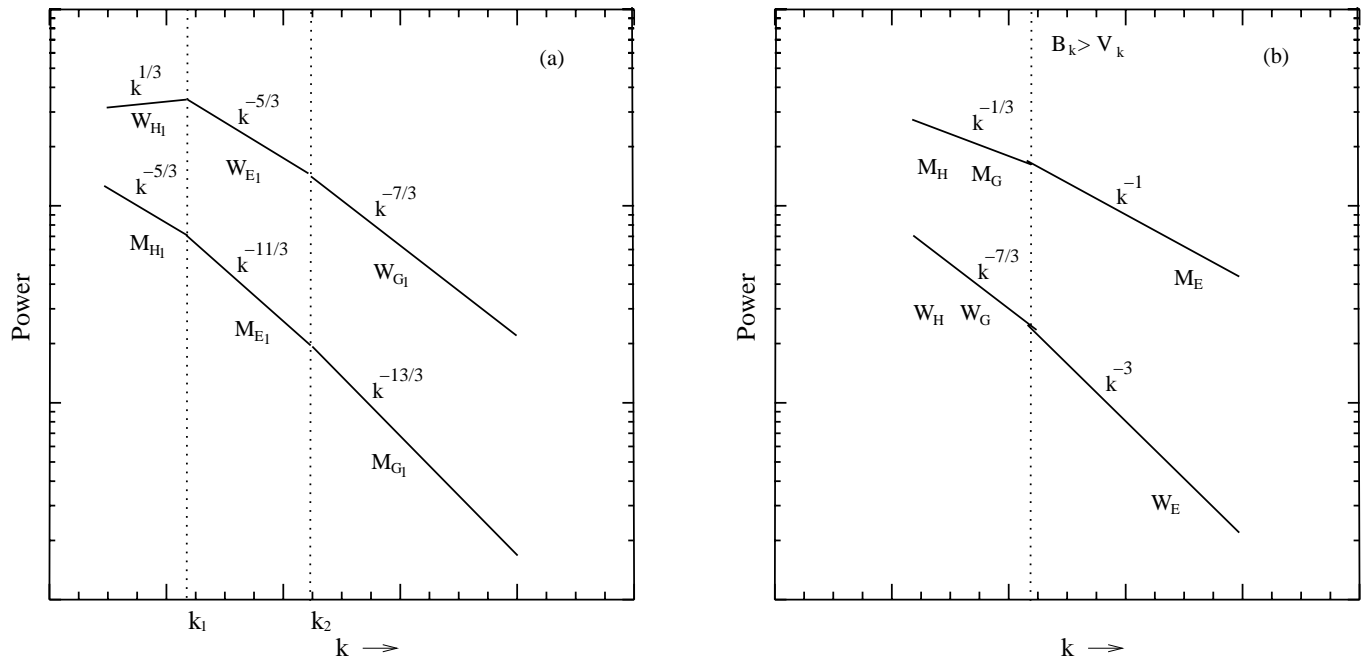

Fig. 1. (a) Schematic Magnetic $(M)$ and Kinetic $(W)$ spectra (Shear-cyclotron mode) for $\alpha=k^{-1}$ in the Hall region ( $\left.k \gg 1\right)$, (b) Schematic Magnetic $(M)$ and Kinetic $(W)$ spectra (Whistler mode) for $\alpha=k$ in Hall region $(k \gg 1)$.

where $\boldsymbol{A}$ is the vector potential. Notice that $H_{G}-H_{M}$ is a combination of the kinetic and the cross helicities.

Notice that the relationship between $v_{k}$ and $b_{k}$ is now $k$ dependent. It is expected, therefore, that the current spectral predictions will be substantially different from those of the standard MHD (where $v_{k}$ and $b_{k}$ have identical spectra) particularly in the range $k \gg 1$, when the Hall term dominates in Eq. (6). The introduction of the Hall term, which brings in an intrinsic scale removes the MHD spectral degeneracy and generates new scale-specific effects.

\section{Spectral energy distributions}

The nonlinear solution is an exact solution for waves propagating in one direction. For the superposition of the right and the left propagating waves the nonlinearity remains and this is what could give rise to the cascading processes as is surmised in the ideal MHD turbulence. The large wavevector limit of the dispersion relation of the nonlinear waves basically leads to the dispersive effects with the difference that now the relation between the velocity and the magnetic field amplitudes is also wave vector dependent. With these qualifying remarks we proceed to derive the spectral energy distributions using the Kolmogorov hypotheses according to which the spectral cascades proceed at a constant rate governed by the eddy turn over time $\left(k \boldsymbol{v}_{k}\right)^{-1}$. For $\varepsilon_{E}$ denoting the constant cascading rate of the total energy $E$, Eq. (35) along with Eq. (22) yields the dimensional equality

$\left(k v_{k}\right)\left[1+(\alpha)^{2}\right] \frac{v_{k}^{2}}{2}=\varepsilon_{E}$.

The omnidirectional spectral distribution function $W_{E}(k)$ (kinetic energy per gram per unit wave vector $v_{k}^{2} / k$ ), then, takes the form

$W_{E}(k)=\left(2 \varepsilon_{E}\right)^{\frac{2}{3}}\left[1+(\alpha)^{2}\right]^{-\frac{2}{3}} k^{-\frac{5}{3}}$.
Consequently, Eq. (22) yields:

$M_{E}(k)=(\alpha)^{2} W_{E}(k)$.

where $M_{E}(k)=b_{k}^{2} / k$ is the similarly defined omnidirectional spectral distribution function of the magnetic energy density.

The cascading of the magnetic helicity $H_{M}\left(\varepsilon_{H}\right.$ being the cascading rate for helicity) produces a different dimensional equality

$\left(k v_{k}\right)\left(0.5 \frac{b_{k}^{2}}{k}\right)=\varepsilon_{H}$

resulting in the following different kinetic and magnetic spectral energy distributions:

$W_{H}(k)=\left(2 \varepsilon_{H}\right)^{\frac{2}{3}}(\alpha)^{\frac{-4}{3}} k^{-1}$,

$M_{H}(k)=(\alpha)^{2} W_{H}(k)$

Finally, the cascading of the generalized helicity with a constant rate $\varepsilon_{G}$ gives

$\left(k v_{k}\right)\left[0.5 g(k) v_{k}^{2}\right]=\varepsilon_{G}$,

$g(k)=(\alpha+k)^{2} k^{-1}$

leading to the spectral energy distributions:

$W_{G}(k)=\left(2 \varepsilon_{G}\right)^{\frac{2}{3}}[g(k)]^{-\frac{2}{3}} k^{-\frac{5}{3}}$,

and

$M_{G}(k)=(\alpha)^{2} W_{G}(k)$. 


\section{Modeling solar wind spectra}

The observed frequency spectra of the solar wind are transformed into the wave vector spectra Doppler shifted by the Super Alfvénic Solar wind flow. Although the anisotropy of the MHD turbulence is now being highly emphasized (Matthaeus et al., 1996), we model the observed reduced omnidirectional spectra with the findings of the isotropic cascade considered in Sect. 3. The incompressible nonlinear solution applies essentially to a plasma with $\beta \rightarrow \infty$ case. The plasma $\beta$ for the solar wind varies from less than to much larger than unity. Here we neglect compressibility effects and highlight the crucial contributions of the Hall effect through the shear Alfvén modes as the steepening of the solar wind spectra appears near the ion inertial scale, a characteristic of the Hall effect. It is not clear from the observations if the density fluctuations also has a characteric scale comparable to the ion inertial scale. It is well known that weak compressibility ( $v \ll$ sound speed) makes a very small change in the Kolmogorov $(-5 / 3)$ spectrum. The kinetic energy spectra is also not well known in this region. In the absence of such information for simplicity we resort to the incompressibility assumption and show that the three spectral distributions derived in Sect. 3 may model the three branch spectrum $\left(k^{-1}\right.$, $k^{-5 / 3}, k^{-\alpha_{1}} \alpha_{1} \simeq 3-4$ ) of the magnetic fluctuations in the solar wind.

If the turbulence is dominated by velocity field fluctuations $\left(\boldsymbol{v}_{k}^{2} \gg \boldsymbol{b}_{k}^{2}\right)$ (which happens, according to Eq. (22), for $(\alpha \ll 1)$, or $(k \gg 1)$ for $\alpha \simeq\left(k^{-1}\right)$, the spectral expressions under the joint dominance of the Hall term and the velocity fluctuations $(k \gg 1)$ simplify to (Fig. 1)

$$
\begin{aligned}
& W_{E_{1}}(k)=\left(2 \varepsilon_{E}\right)^{2 / 3} k^{-5 / 3}, \quad M_{E_{1}}(k)=\left(2 \varepsilon_{E}\right)^{2 / 3} k^{-11 / 3} \\
& W_{H_{1}}(k)=\left(2 \varepsilon_{H}\right)^{2 / 3} k^{1 / 3}, \quad M_{H_{1}}(k)=\left(2 \varepsilon_{H}\right)^{2 / 3} k^{-5 / 3} \\
& W_{G_{1}}(k)=\left(2 \varepsilon_{G}\right)^{2 / 3} k^{-7 / 3}, \quad M_{G_{1}}(k)=\left(2 \varepsilon_{G}\right)^{2 / 3} k^{-13 / 3} .
\end{aligned}
$$

In the case wherein $\alpha=1$ for $k \ll 1$, one obtains the standard Alfvénic state with $v_{k} \propto b_{k}$, and the corresponding spectra (Fig. 2) are (suffix 1 is used for the Hall Dominant and 2 for the standard MHD limit):

$$
\begin{aligned}
& M(k)=W(k) \\
& W_{E_{2}}(k)=\left(2 \varepsilon_{E}\right)^{2 / 3} k^{-5 / 3} \\
& W_{H_{2}}(k)=\left(2 \varepsilon_{H}\right)^{2 / 3} k^{-1} \\
& W_{G_{2}}(k)=\left(2 \varepsilon_{G}\right)^{2 / 3} k^{-1}
\end{aligned}
$$

For the second root of $\alpha \simeq k, k \gg 1$, representing the $\beta \rightarrow \infty$ limit of the whistler type fluctuations, we find the following spectra:

$$
\begin{aligned}
& W_{E_{w}}(k)=\left(2 \varepsilon_{E}\right)^{2 / 3} k^{-3}, \quad M_{E_{w}}(k)=\left(2 \varepsilon_{E}\right)^{2 / 3} k^{-1} \\
& W_{H_{w}}(k)=\left(2 \varepsilon_{H}\right)^{2 / 3} k^{-7 / 3}, \quad M_{H_{w}}(k)=\left(2 \varepsilon_{H}\right)^{2 / 3} k^{-1 / 3} \\
& W_{G_{w}}(k)=\left(2 \varepsilon_{G}\right)^{2 / 3} k^{-7 / 3}, \quad M_{G_{w}}(k)=\left(2 \varepsilon_{G}\right)^{2 / 3} k^{-1 / 3} .
\end{aligned}
$$

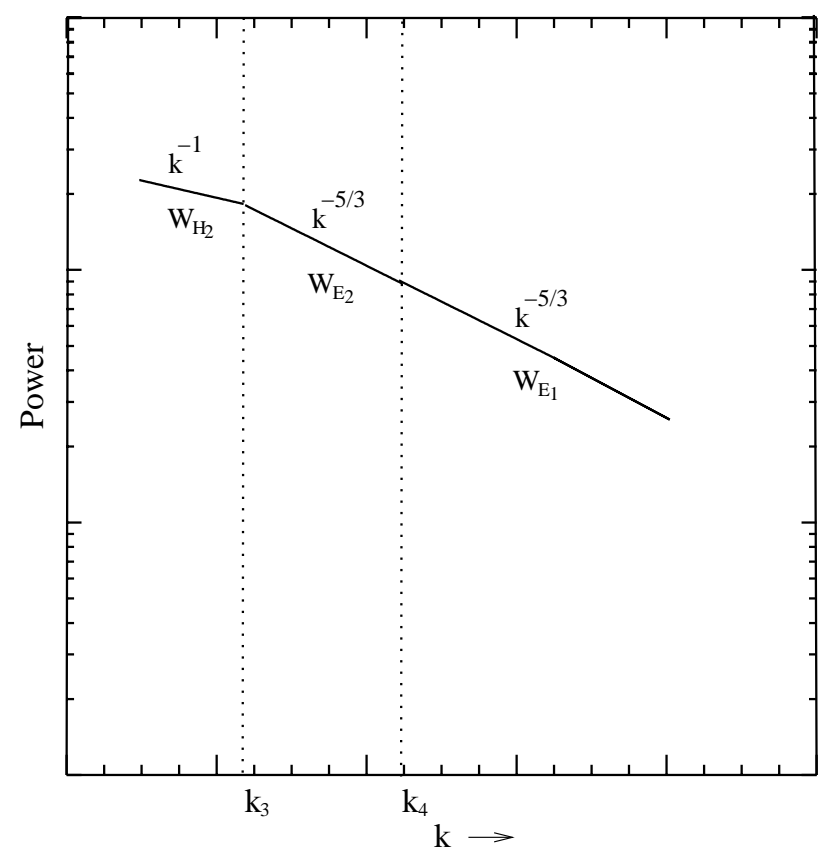

Fig. 2. Schematic Magnetic $(M)$ and Kinetic $(W \equiv M)$ spectra (shear-Alfvén mode) for $\alpha \simeq 1$ in the Alfvén region $(k \ll 1)$.

The observed solar wind magnetic spectrum will be generated if we were to string together the three branches $M_{E_{1}}(k)\left(\propto k^{-11 / 3}\right), M_{H_{1}}(k)\left(\propto k^{-5 / 3}\right)$, and $M_{H_{2}}(k)\left(\propto k^{-1}\right)$. The rationale as well as the modality for stringing different branches originates in the hypothesis of selective dissipation. It was, first, invoked in the studies of two-dimensional hydrodynamic turbulence (Fjortoft, 1953; Hasegawa, 1985). In 2-D hydrodynamic turbulence, for instance, the enstrophy invariant, because of its stronger $k$ dependence (and hence larger dissipation) compared to the energy invariant, dictates the large $k$ spectral behavior. Therefore, the entire inertial range spectrum has two segments - the energy dominated low $k\left(\propto k^{-5 / 3}\right)$, and the enstrophy dominated high $k\left(\propto k^{-3}\right)$. The procedure amounts to placing the spectrum with the highest negative exponent $\left(\propto k^{-13 / 3}\right)$ at the highest $k$-end,then the spectrum with the next lower negative exponent $\left(\propto k^{-11 / 3}\right)$ and finally the one with the lowest negative exponent of $k$ at the lowest $k$-end (e.g. Fig. 1a).

Notice that the observed solar wind magnetic spectra consisting of the branches $k^{-\alpha_{1}}\left(\alpha_{1} \sim 3-4\right), k^{-5 / 3}$ and $k^{-1}$ can be reproduced by stringing the Hall state spectral branches at large $k$ with Alfvénic state branches at small $k$; The result is displayed in Fig. 3. This is rather fortunate because in HMHD it is precisely for large $k$ that the Hall term is dominant while for small $k$, the standard Alfvénic behavior prevails. Within the framework of this dimensional Kolmogorov-inspired model, there is another consistent way of constructing the observed magnetic spectrum of the solar wind from the spectral relations we derived as shown in Fig. 4. The difference lies in the positioning of the overlap between the Hall and the Alfvén states. 


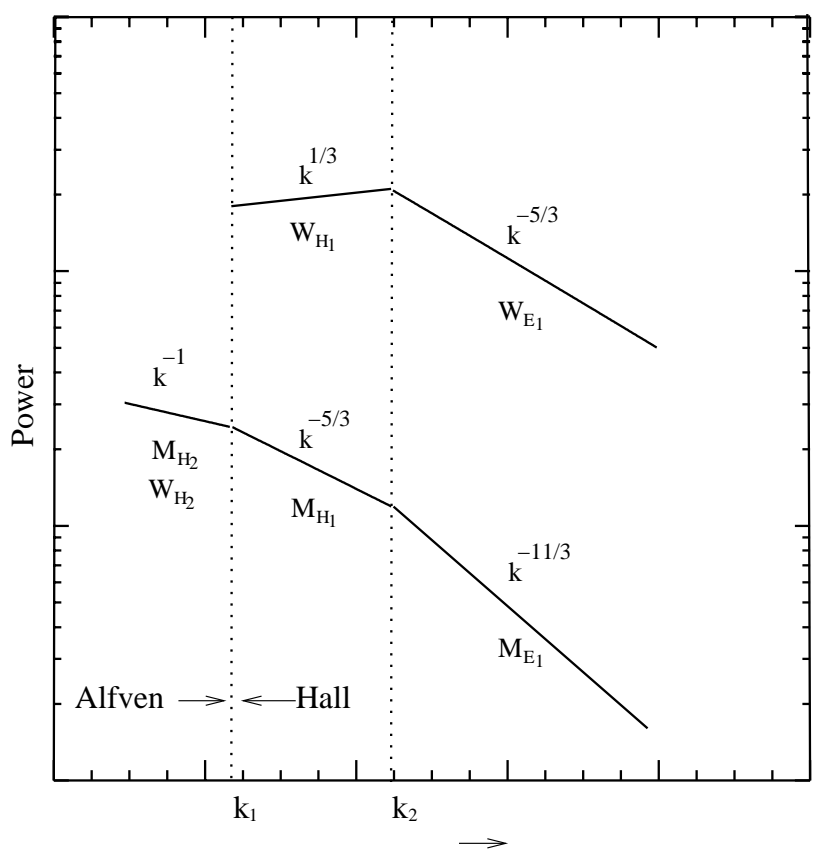

Fig. 3. Modeled Magnetic $\left(M_{1}\right)$ spectra along with the corresponding Kinetic $\left(W_{1}\right)$ spectra.

\section{Hall time scale}

From the magnetic induction equation

$\frac{\partial}{\partial t} \boldsymbol{B}=-c \nabla \times \boldsymbol{E}=\nabla \times\left(\boldsymbol{V}_{i}-\boldsymbol{J}\right) \times \boldsymbol{B}$

one can identify a characteristic Hall time scale

$\tau_{H}=\left[k\left(V_{i}+V_{H}\right)\right]^{-1}$

where $V_{H}=-J$ is the hall velocity. Thus $\tau_{H}=\left[k\left(v_{k}+k b_{k}\right)\right]^{-1}=\left[k\left(v_{k}+k \alpha v_{k}\right)\right]^{-1}=\left(2 k v_{k}\right)^{-1}$ for $b_{k}=\alpha v_{k}$ and $\alpha=k^{-1}$.

Thus the Hall time scale is half the hydrodynamic time scale for Hall shear Alfvénic fluctuations. This is a further confirmation that the combination of the hydrodynamic time scale along with the association of fluctuations with the shear Alfvén waves is the right choice for reproducing the observed spectra.

\section{Dissipative and dispersive attempts}

The diffusion equation for the omnidirectional spectral density $W$ can be written as (Li et al., 2001):

$\frac{\partial W}{\partial \tau}=\frac{\partial}{\partial k}\left[k^{2} D \frac{\partial\left(k^{-2} W\right)}{\partial k}\right]+\gamma W+S$

For a power law form:

$W(k)=W_{0} k^{-s}$

In the steady state, in the absence of the source $S$ and dissipation or growth $\gamma$, the diffusion coefficient $D \propto k^{1+s}$. For $s=3$,

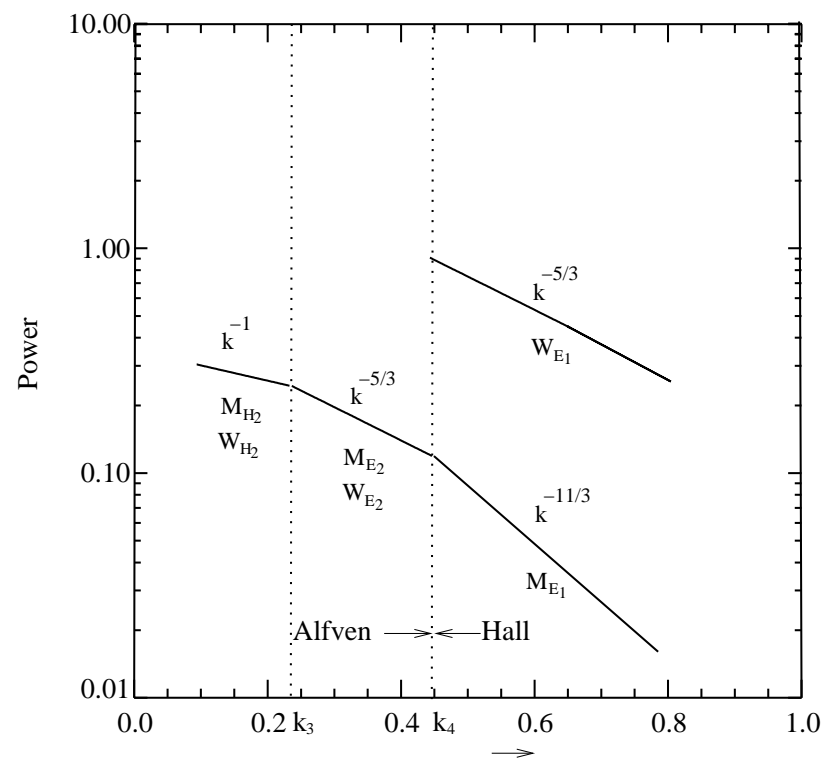

Fig. 4. Modeled Magnetic $\left(M_{2}\right)$ spectra along with the corresponding Kinetic $\left(W_{2}\right)$ spectra.

$D \propto k^{4}$. This is achieved by defining $D=k^{2} / \tau$ and identifying $\tau$ with the inverse of the frequency of the whistler mode so that $\tau \approx \omega^{-1} \propto\left(k+k^{2}\right)^{-1}$, leading to the so called dispersion range of the spectrum for $s=3$ (Stawicki et al., 2001). We wish to comment that identifying the cascade time $\tau$ with the wave period is not correct since the cascade is a nonlinear process and its time scale should be a function of the fluctuation amplitude.

The attempts to account for the steepened spectrum by invoking dissipation fail (Gary, 1999; Li et al., 2001) as can be seen from the following considerations. The diffusion equation for the omnidirectional spectral density $W$ in the steady state with dissipation and no source can be written as:

$\frac{\partial}{\partial k}\left[k^{2} D \frac{\partial\left(k^{-2} W\right)}{\partial k}\right]+\gamma W=0$.

With $D=k^{2} / \tau \approx k^{2}\left(k v_{k}\right)=k^{3}[k W(k)]^{0.5} \propto k^{(7-s) / 2}$ we find $\gamma \propto k^{(3-s) / 2}$. Thus for the steepened spectrum with $s=3$, $\gamma \propto k^{0}$ and for $s=4, \gamma \propto k^{-0.5}$. But the damping of the Alfvén waves by the ion- cyclotron resonance absorption has an exponential dependence on $k$ and the damping of the magnetosonic waves by Landau resonance has a power law dependence much stronger than $k^{-0.5}$. Thus the dissipation processes are inadequate to account for the steepened part of the solar wind spectrum.

\section{Discussion and conclusions}

We have been able to reproduce the observed magnetic spectrum of the Solar wind by including the physics of the Hall current and the fluid vorticity in two-fluid magnetohydrodynamics. The steepened part of the spectrum is shown to arise in the inertial range as contrasted with the dissipative 
and dispersive ranges invoked in some earlier studies. The observed turbulent fluctuations are shown to be associated with Hall shear Alfvén waves cascading with the hydrodynamic timescale also the Hall timescale. The scale dependent relationship between the velocity and the magnetic fluctuations redefines the concepts of the equipartition of energy and the Alfvén ratio. These along with the kinetic energy spectrum offer further possibilities of validation of this exact nonlinear incompressible HMHD model of turbulence. The compressibility effects which will in general not permit an exact solution have to be necessarily studied in a linearized version of HMHD.

Acknowledgements. The authors gratefully acknowledge the support from the Abdus Salam International Center for Theoretical Physics, Italy where a part of the work was done. The authors thank B. Varghese for his help in the preparation of this manuscript. The authors thank the referees for their critical comments and helpful suggestions

Edited by: T. Passot

Reviewed by: two referees

\section{References}

Behannon, K. W.: Heliocentric distance dependence of the interplanetary magnetic field, Reviews of Geophysics and Space Physics, 16, 125-145, 1978.

Biermann, L.: Komentenschweife und solare korpuskularstrahlung, Z. Astrophys., 29, 274-286, 1951.

Chapman, S.: On outline of a theory of magnetic storms, Proc. Roy. Soc. A, 95, 61-83, 1919.

Coleman, P.J. Jr.,: Turbulence, viscosity, and dissipation in the Solar wind plasma, Astrophys. J., 153, 371-388, 1968.

Cranmer, S. R. and von Ballagooijen, A. A.: Alfvénic turbulence in theextended solar corona: kinetic effects and proton heating, Astrophys. J., 594, 573-591, 2003.
Denskat, K. U., Beinroth, H. J., and Neubauer, F. M.: Interplanetary magnetic field power spectra with frequencies from $2.4 \times 10^{-5} \mathrm{~Hz}$ to $470 \mathrm{~Hz}$ from HELIOS-observations during solar minimum conditions, J. Geophys., 54, 60, 1983.

Gary, S. P.: Collisionless dissipation wavenumber: Linear theory, J. Geophys. Res., 104, 6759-6762, 1999.

Ghosh, S., Siregar, E., Roberts, D. A., and Goldstein, M. L.: Simulation of high-frequency solar wind power spectra using Hall magnetohydrodynamics, J. Geophys. Res., 101, 2493-2504, 1996.

Goldstein, M. L., Roberts, D. A., and Fitch, C. A.: Properties of the fluctuating helicity in the inertial and dissipation ranges of solar wind turbulence, J. Geophys. Res., 99, 11 519-11 538, 1994.

Goldstein, M. L., Roberts, D. A., and Matthaeus, W. H.: Magnetohydrodynamic turbulence in the solar wind, Annual Rev. Astron. Astrophys., 33, 283-325, 1995.

Hasegawa, A.: Self-organization processes in continuous media, Adv. Phys., 34, 1-42, 1985.

Krishan, V. and Mahajan, S. M.: Hall-MHD turbulence in solar atmosphere, Solar Physics, 220, 29-41, 2004.

Leamon, R. J., Smith, C. W., Ness, N. F., Matthaeus, W. H., and Wong, H. K.: Observational constraints on the dynamics of the interplanetary magnetic field dissipation range, J. Geophys. Res., 103, 4775-4787, 1998.

Li, H., Gary, P., and Stawicki, O.: On the dissipation of magnetic fluctuations in the Solar wind, Geophys. Res. Lett., 28, 13471350, 2001.

Mahajan, S.M. and Yoshida, Z.: Double Curl Beltrami Flow: diamagnetic structures, Phys. Rev. Lett., 81, 4863-4866, 1998.

Marsch, E.: MHD turbulence in the solar wind, in Physics of the Inner Heliosphere II, Particles, Waves and Turbulence, edited by Schwenn, R. and Marsch, E., Springer-Verlag, New York, 1991.

Matthaeus, W. H., Ghosh, S., Oughton, S., and Roberts, D. A.: Anisotropic three-dimensionsl MHD turbulence, J. Geophys. Res., 101, 7619-7629, 1996.

Parker, E. N.: Dynamics of the interplanetary gas and magnetic fields, Astrophys. J., 128, 664-676, 1958.

Stawicki, O., Gary, P. S., and Li, H.: Solar wind magnetic fluctuation spectra: dispersion versus damping, J. Geophys. Res., 106, 8273-8281, 2001. 